\title{
Genomic profiling of sinonasal squamous cell carcinoma.
}

Fernando López, MSc ${ }^{1}$; José L. Llorente, MD, $\mathrm{PhD}^{1}$; Cristina García-Inclán, MSc ${ }^{1}$, Marta Alonso-Guervós, $\mathrm{PhD}^{1}$; Mari Paz Cuesta-Albalad, MSc${ }^{1}$; Manuel Florentino Fresno, MD, $\mathrm{PhD}^{2}$; César Álvarez-Marcos, MD, $\mathrm{PhD}^{1}$; Carlos Suárez, MD, $\mathrm{PhD}^{1}$; Mario A. Hermsen, PhD ${ }^{1}$

${ }^{1}$ Dept of Otolaryngology. IUOPA. Hospital Universitario Central de Asturias, Oviedo, Asturias, Spain.

${ }^{2}$ Dept of Pathology. Hospital Universitario Central de Asturias, Oviedo, Asturias, Spain.

Acknowledgements: This study was supported by grants IB05-115 of Fundación para el Fomento en Asturias de la Investigación Científica Aplicada y la Tecnología (FICYT), PI051387 and EMER07-048 of Fondos de Investigación Sanitaria (FIS), and RD06/0020/0034 of Red Temática de Investigación Cooperativa en Cáncer (RTICC), Spain. The authors thank Eva Allonca and Sira Potes for their technical support, and Bauke Ylstra of the Microarray Facility of the VUmc Medical Center, Amsterdam.

\section{Corresponding autor:}

Mario A. Hermsen

Dept. Otolaryngology

Instituto Universitario de Oncología del Principado de Asturias (IUOPA)

Edificio H Covadonga 1 a Planta Centro, lab 2

Hospital Universitario Central de Asturias

Celestino Villamil s/n, 33006 Oviedo

SPAIN

Tel: 34985 107956; Fax: 34985108015

email: mhermsen@hca.es

Running Title: Genomic profiling of sinonasal carcinoma

Key Words: maxillary sinus, ethmoid sinus, squamous cell carcinoma, microarray CGH, MLPA. 


\section{ABSTRACT}

Background. Sinonasal squamous cell carcinomas (SNSCC) are rare tumors with no etiological link to tobacco and alcohol, as opposed to other squamous cell carcinomas of the head and neck (HNSCC). Little is known about the genetic changes in SNSCC.

Methods. DNA copy number changes of SNSCC were analyzed by MLPA (multiplex ligationdependent probe amplification) and microarray comparative genomic hybridization (maCGH), and results were related to clinico-pathological features.

Results. Copy number losses most frequently included genes at 9p21, 13q14, 17p13, 17q21 and 18q11. Frequent gains were observed on 8q24, 11q13, 17q12, 19p13 and 20q11-q13.

Conclusion. The genomic profile of SNSCC showed a number of chromosomal regions with copy number changes similar to those known in HNSCC, in spite of the differences in etiology. 


\section{INTRODUCTION}

Sinonasal squamous cell carcinomas (SNSCC) are malignant epithelial tumors originating in the respiratory mucosa of the nasal cavities and paranasal sinuses. A wide variety of histological types of tumors originates in this anatomical area, mostly epithelial (over $75 \%$ ), of which the most common is squamous cell carcinoma, accounting for $80-90 \%$ of all nasal tumors $^{1-3}$. SNSCC are located in a complex anatomical area, close to structures such as the eyes and brain, which is of special relevance for surgery and postoperative treatment, since mutilation and aesthetic deformities are difficult to avoid ${ }^{4}$. Despite improvements in the field of surgery and radiotherapy, patients with these tumors still face a very unfavorable prognosis. In Spain, the incidence is less than 1 case per 100.000 inhabitants per year, occurring predominately among men with a mean age of presentation of 50 to 60 years ${ }^{2,5}$. Its most frequent origin is the nasal cavity, followed by the maxillary sinus, although these numbers are reversed in some publications ${ }^{1,3}$.

The clinical presentation of patients with SNSCC is very insidious and tumors are often diagnosed at advanced stages. Affected lymph nodes at diagnosis are uncommon, as well as the presence of distant metastases. The overall prognosis is poor with an overall 5 year survival of $30-50 \%$; local recurrence is the main cause of death among patients ${ }^{4,6,7}$. Prognosis of SNSCC of the nasal cavities is better than SNSCC of the sinuses due to its diagnosis at earlier stages. Advanced T stage, skull base involvement, orbital extension, and local recurrence are predictors of poor survival. The standard therapeutic modality is surgery, followed by radiotherapy in advanced stages. In some cases chemotherapy is used ${ }^{4,6-9}$.

Little is known about the molecular pathways of SNSCC development. Occupational exposure to leather and wood dust as well as several chemicals as nickel, arsenic and formaldehyde have been implicated in the etiology of SNSCC ${ }^{10-12}$. Tobacco however, a strong etiological factor for the majority of head and neck squamous carcinomas (HNSCC), does not play an important role in SNSCC ${ }^{13,14}$. Until the present moment, genetic studies on SNSCC have demonstrated TP53 mutations and p53 overexpression in up to 56\% of cases $^{15-17}$. Kras mutations have been reported, but only in $1 \%$ of $\operatorname{cases}^{18}$. Bandoh et al. 
suggested that TP53 mutations and a low apoptotic index are associated with poor survival ${ }^{16}$. COX2 expression was observed in low frequency, which may rule out a possible role for chronic inflammation in SNSCC development ${ }^{17}$. Using loss of heterozygosity analysis, Götte et al. have shown losses on chromosomes $3 p, 8 p, 17 p$ and $18 q^{19}$. There are very few genome-wide studies on genetic changes in SNSCC. Cytogenetic analyses on small numbers of cases revealed complex karyotypes with structural aberrations involving many chromosomes. Losses were detected on $6 q$ and gains on $3 q$ and $11 q^{20,21}$.

We have performed a genome-wide screening of copy number abnormalities by multiplex ligation-dependent probe amplification (MLPA) and microarray comparative genome hybridization (maCGH) on a series of 27 SNSCC and investigated possible relations between genetic results and the clinical and pathological characteristics.

\section{MATERIAL AND METHODS}

\section{Tumor Specimens}

Tissue samples of 27 squamous cell carcinomas of nasal cavity and paranasal sinuses were obtained from previously untreated patients treated at the Department of Otolaryngology, Hospital Universitario Central de Asturias, between 1989 and 2006, through radical surgery. Patients with primary tumors in the nasal vestibule or septum were excluded, because these tumors probably arise from the skin rather than nasal respiratory mucosa. No patient had had professional exposure to leather or wood dust or industrial chemicals. Of 27 primary tumor specimens, 20 were obtained from paraffin blocks and seven from frozen tumor samples. All patients gave their signed informed consent and the study had received prior approval from the ethical committee of our institution. 


\section{Clinical Variables}

Of the 27 SNSCC patients, 17 were male (63\%) and 10 female (37\%). The mean age was 65 years (range 47-85). Twenty-three tumors were located in the maxillary sinus (85\%) and 4 in the ethmoid sinus (15\%). Our series comprised 10 well-differentiated tumors (37\%), 6 moderately-differentiated (22\%) and 11 poorly-differentiated (40\%). According to the T stage of the TNM classification (UICC TNM classification of malignant tumors) ${ }^{22}$, our series consisted of 3 tumors T2 (11\%), 8 tumors T3 (30\%), 10 tumors T4a (37\%) and 6 tumors T4b $(22 \%)$. If classifying by stage the series consists of 3 tumors in stage II (11\%), 8 tumors in stage III (30\%), 7 tumors in stage IVa (26\%) and 9 tumors in stage IVb (33\%). At the time of diagnosis 4 patients had affected lymph nodes (15\%) and no patient had distant metastases. The periorbita was affected in 5 patients (18,5\%) and another $5(18,5 \%)$ had clear orbital invasion. One patient (4\%) had intracranial involvement at diagnosis. Nineteen patients (70\%) received radiotherapy after radical surgery. The median follow-up time was 16 months (1-211). A detailed description of patient and tumor characteristics is given in table 1.

\section{DNA extraction}

Tumor DNA was extracted from frozen tissue samples and from paraffin-embedded tissue samples using Qiagen extraction kits (Qiagen $\mathrm{GmbH}$, Hilden, Germany) according to the manufacturer's recommendations. MLPA on DNA from frozen tumor tissue was analyzed by comparing to normal reference DNA extracted from blood of healthy donors and MLPA on DNA from paraffin tumor tissue was analyzed by comparing to normal reference DNA obtained from paraffin blocks of normal tissue.

Special care was taken to obtain high quality DNA from the formaldehyde-fixed, paraffin-embedded tissues. DNA extracted from archival material can be partly degraded and cross-linked, the extent of which depends on the $\mathrm{pH}$ of the formaldehyde and the time of the fixation before paraffin embedding. To improve the quality of the isolated DNA, we have applied an elaborate extraction protocol especially for paraffin tissues, which includes thorough deparaffination with xylene, methanol washings to remove all traces of the xylene, 
and a 24 -hour incubation in $1 \mathrm{~mol} / \mathrm{L}$ sodium thiocyanate to reduce cross-links. Subsequently, the tissue pellet is dried and digested for 3 days in lysis buffer with high doses of proteinase $\mathrm{K}$ (final concentration $2 \mathrm{ug} / \mathrm{uL}$, freshly added twice a day). With this protocol, most formaldehyde-fixed, paraffin-embedded tissue samples yielded DNA of relatively good quality, with A260/A280 values between 1,7 and 2.0 measured by Nanodrop (Thermo Scientific, Wilmington DE, USA) and lengths between 2000 and 20,000 bp, and these samples were used in MLPA analysis. For microarray CGH, a technique more sensitive to DNA quality, we performed an additional quality test using the ENZO Bioscore Screening and Amplification kit (Enzo Life Sciences, Lörrach, Germany). The assay consists of an isothermal whole genome amplification reaction using $100 \mathrm{ng}$ of DNA, followed by a purification by QIAquick PCR Purification columns (Qiagen $\mathrm{GmbH}$, Hilden, Germany) and measurement of the DNA concentration by Nanodrop ((Thermo Scientific, Wilmington DE, USA). Only those samples that gave a total yield of 3.0 or more were used for microarray $\mathrm{CGH}$ analysis.

\section{MLPA}

The MLPA technique has been performed as described in detail previously ${ }^{23}$. Two different kits were used, together analysing 58 cancer related genes distributed throughout the genome (kit P005 and P084, MRC-Holland, Amsterdam, the Netherlands). Each probe is composed of two parts that hybridize to adjacent target sequences in the DNA. After a ligation step and a PCR amplification, each probe gives rise to a product with a unique size between 130 and $480 \mathrm{bp}$. Briefly, $100 \mathrm{ng}$ DNA was denatured at $98^{\circ} \mathrm{C}$ for 5 min and hybridized with the MLPA probe mixture at $60^{\circ} \mathrm{C}$ for 16 hours. Ligation of the two parts of each probe was performed by a thermostable ligase. All probe ligation products have the same end sequences and were amplified by PCR using the same primer pair for $60^{\circ} \mathrm{C} 1 \mathrm{~min}$, 33 cycles of $95^{\circ} \mathrm{C} 30 \mathrm{sec}, 60^{\circ} \mathrm{C} 30 \mathrm{sec}$ and $72^{\circ} \mathrm{C} 1 \mathrm{~min}$, followed by $20 \mathrm{~min}$ at $72^{\circ} \mathrm{C}$ and kept cold at $4^{\circ} \mathrm{C}$. The products were subsequently analyzed on an ABI Prism 3100 sequencer and by GeneScan v3.7 software (Applied Biosystems, Warrington, UK). 
Fourteen control experiments using normal DNA from 7 different donors were used to calculate median reference values and the standard deviations for every probe. Each tumor sample was analyzed at least twice. For every gene, the relative copy number was calculated by dividing the average relative peak area of the tumor by the median relative peak area of the normal reference samples. We observed that a MLPA experiment analysing a new normal DNA resulted in relative copy numbers varying between 0.85 and 1.15 , including the standard deviation, for each probe. Therefore we decided that relative copy number values, including the standard deviation, lower than 0.8 were interpreted as losses, higher than 1.2 as gains and 2.0 or higher as amplifications.

\section{Microarray CGH}

MaCGH was performed on 7 fresh samples and 4 paraffin-embedded samples with a good quality of DNA as described previously ${ }^{24}$. Briefly, sample DNA and reference DNA (extracted and pooled from blood of 10 different healthy donors) were differently labeled by random priming. Three hundred nanograms test and $300 \mathrm{ng}$ reference DNA were hybridized to a 30.000-oligonucleotide array printed on Codelink activated slides (Amersham Biosciences, Barcelona, Spain). This array contained 29.134 oligos covering 28.830 unique genes. Hybridization and washing took place for two nights in a specialized hybridization chamber (GeneTAC/HybArray12 hybstation [Genomic Solutions/Perkin Elmer, Waltham, MA]). Images were acquired using a Microarray scanner G2505B (Agilent Technologies, Amstelveen, Netherlands). Analysis and data extraction were quantified by BlueFuse (BlueGnome, Cambridge, UK). The pooled reference DNA served as an internal control of quality. Normalization of the calculated ratios was done against the mode of all ratios. Graphics were plotted using a moving average of log2 ratios of 5 neighboring clones. Gains and losses were defined as at least in 2 neighboring clones with deviations of 0.2 or more from log2 ratio = 0.0. High-level amplification was considered when at least 2 neighboring clones reached a $\log 2$ ratio of 1.0 or higher. 


\section{Statistical Analysis}

Possible correlations between genetic and clinical parameters were statistically analyzed by SPSS 12.0 software for Windows (SPSS® Inc. Illinois, USA), using the Fisher exact Chi2 test. Kaplan-Meier analysis was performed for estimation of survival, comparing distributions of survival through the logarithmic range test (log-rank test). $P$ values below 0.05 were considered significant.

\section{RESULTS}

\section{Clinical data}

During the time of follow up, seventeen patients developed local recurrence (62\%) and 5 patients developed distant metastases (18\%). At the time of writing this manuscript, a total of 9 patients remained disease-free. The overall 5 year survival was $28 \%$ and the median disease-free survival was 9 months (range 1-93). We found no significant relation between the overall or disease-free survival and clinical parameters such as localization, histological differentiation, tumor stage, or disruption of the orbit. Intracranial invasion and affected lymph nodes at the time of diagnosis were observed in cases with poor clinical outcome. The main causes of death in our series were recurrences and distant metastases.

\section{Genetic data}

Both MLPA and maCGH analysis showed genetic alterations in all cases studied. Examples of a MLPA and a maCGH result is given in figures 1 and 2, respectively. The most recurrent alterations detected by MLPA were losses of BRCA1 and CDH2 (38\%), CRK (33\%), STCH and RB1 (29\%), CDKN2A and IL18 (25\%), and IL4 and BCAR (21\%); and gains of EMS1 and BCL2L1 (37\%), RECQL4, RELA and ERBB2 (29\%), CCND1 (26\%), PTPN1 (22\%) and MYC (21\%). The chromosomal regions where these genes are localized were also most frequently observed by maCGH, which indicated additional hotspots of losses at $2 q 25,3 p 14$, 4q21-qter and 9q13, and gains at 5p15, 6p21, 7p12 and 16p13. All DNA copy number gains and losses identified by MLPA and maCGH are graphically presented in figures 3 and 4 
respectively. High level amplifications occurring in two or more of the 27 cases were detected by MLPA for EMS1 and CCND1 (11q13, 4 cases), and ERBB2 (17q21, 2 cases). All were confirmed in at least one of the 11 cases analyzed by maCGH. Table 2 shows the complete list of amplifications observed both by microarray $\mathrm{CGH}$ and by MLPA. Microarray CGH detected two other recurring amplicons, at $7 \mathrm{p} 12$ ( 3 cases) and at $11 \mathrm{p} 13$ ( 2 cases). Candidate genes in these localizations are EGFR and CD44 respectively. Unfortunately, these two genes were not included in the 58 genes studied by MLPA.

\section{Clinical-genetical correlations}

The four cases with lymph node metastasis at the time of diagnosis showed more complex copy number changes, with virtually all chromosomes involved in gains and losses, and with several high level amplifications (see maCGH result of case number 26, figure 2). Five out of eight tumors that developed either lymph node or distant metastasis versus one out of nineteen that did not, demonstrated copy number gains of the oncogene PTPN1 at 20q13 (Fisher Exact Chi2 $\mathrm{p}=0.004$ ). To a lesser extent this relation was also seen for gains of oncogenes CCND1 and EMS1 at 11q13 and PTP4A3 at 8q24 (Table 3). However, neither of these genetic alterations correlated to recurrence or survival. In fact, no single copy number abnormality was associated with overall or disease-free survival as evaluated by Kaplan Meier analysis. 


\section{DISCUSSION}

Little is known about the genetic changes involved in the development of SNSCC. To our knowledge, this article is the first to perform a comprehensive, genome-wide study of DNA copy number changes using two different techniques in SNSCC. The number of tumors in this study may be low, but considering that SNSCC is a rare malignancy we believe our results may serve as a starting point in the study of the molecular biology of SNSCC.

The epidemiological, clinical and pathological variables studied in 27 patients are similar to those described in previous series published in the literature $e^{2,4,6,7}$. The overall survival rate of patients in our series was $28 \%$ after 5 years, similar to the western published series $^{4,6,7}$. However, a Japanese study reported a 5 -year survival of $67 \%$ in T4 tumors ${ }^{9}$. Lymph node metastasis at the time of diagnosis and intracranial invasion decreased the survival significantly, being the most important prognostic clinical factor in SNSCC. As claimed in previous studies, local recurrences and metastases were the main cause of deaths and postoperative radiotherapy increased survival significantly.

The most common malignancy in the head and neck area is squamous cell carcinoma which is strongly associated with exposure to carcinogens such as alcohol and tobacco. Most of these tumors are localized in the larynx, pharynx and oral cavity; the majority of genetic studies concerned these localizations. Califano et al. proposed a genetic model of tumor progression in which normal mucosa develops, via a premalignant stage, into invasive carcinoma, accumulating early chromosomal losses at chromosome arms $3 p$ and $9 p$ and subsequently $17 p$ and more ${ }^{25}$. Genome-wide conventional and microarray CGH studies analysing series of invasive carcinomas have reported frequent losses at $1 p, 3 p, 8 p, 9 p$, $11 q$ ter, 17p, 18q, and gains at 3q, 5p, 7p, 7q, 8q, 11q13 and 20q. Recurring amplifications in descending frequency occurred at $11 q 13,3 q 26$-qter, $7 p 12,8 q 24$ and $8 p 12^{26-28}$.

In contrast to these tumors, SNSCC are not related to tobacco, but to occupational exposure to wood or leather dust, nickel, arsenic and formaldehyde. In addition, premalignant lesions have not been described, although it has been claimed that inverted papillomas may be precursor of SNSCC ${ }^{29}$. Therefore, we expected SNSCC to have a pattern of DNA copy number abnormalities different from HNSCC. However, our combined microarray $\mathrm{CGH}$ and MLPA results demonstrated a number of chromosomal regions with copy number abnormalities similar to HNSCC, such as losses at 1p, 3p, 9p, 11qter, 17p and $18 q$, gains at $3 q, 5 p, 7 p, 8 q, 11 q 13$ and $20 q$, and also high level amplifications at $11 q 13$ (CCND1 and EMS1), 7p12 (EGFR), and 11p13 (CD44) have been described before in HNSCC $^{27,28}$. Two studies concerning the relation between SNSCC and inverted papilloma as possible precursor lesion also suggested that SNSCC genetically resemble HNSCC. Caruana et al. found that HPV is infrequent in SNSCC as opposed to SCC that originated in an inverted papilloma ${ }^{30}$. HPV is also infrequent in HNSCC with the exception of tonsillar 
tumors. Califano and coworkers demonstrated a pattern of LOH in SNSCC similar to HNSCC, while inverted papilloma did not show any $\mathrm{LOH}^{31}$. Overall, it would seem that the histological type of the tumor, squamous cell carcinoma, is more relevant to the pattern of chromosomal gains and losses than etiological factors involved in tumorigenesis. This idea is further supported by the study of Singh et al, who compared chromosomal aberrations of HNSCC from smokers and non-smokers but found no significant differences ${ }^{32}$.

Although concerning only 27 cases, univariate statistical analysis of genetic findings to clinical parameters revealed a significant correlation between gain of PTPN1 at 20q13.2 and tumor dissemination, i.e. tumors that developed either lymph node or distant metastasis. PTPN1 (or PTP1B) is a nonreceptor protein-tyrosine phosphatase involved in many cellular signalling pathways, including EGFR and Ras/ERK ${ }^{33}$. It may function as a suppressor but also as a promotor of neoplastic transformation. Gene amplification and protein overexpression in up to $72 \%$ of cases have been reported in breast cancer, especially in Her2/neu positive tumors ${ }^{33}$. The $20 q 13$ chromosomal region, however, is very gene-rich and there may be other candidate genes explaining the relation to metastasis. Gains and amplifications of the $20 q 13.2$ region have been correlated with lymph node status in gastric cancer $^{34}$, to recurrence in urothelial tumors ${ }^{35}$ and to tumor progression in colorectal carcinoma ${ }^{36}$. This preliminary finding needs to be confirmed in studies with a larger number of cases.

In conclusion, we have shown that SNSCC have a number of genetic changes in common with HNSCC, in spite of differences in etiology. This may be important with regard to clinical decision-making and possibilities for new therapies, such as those directed against EGFR $^{37}$, because an example could be taken from the more prevalent and far more studied HNSCC. 


\section{REFERENCES.}

1. Shah JP, Patel SG. Cavidad nasal y senos paranasales. In: Shah J editor. Cirugía y Oncología de Cabeza y Cuello. Madrid: Elsevier, 2004:57-93.

2. Gras Cabrerizo JR, Sarandeses García A, Montserrat i Gili JR, Orús Dotú C. Revisión de los carcinomas de senos paranasales. Acta Otorrinolaringol Esp. 2007;58:266-275.

3. Suárez Nieto C, Llorente Pendás JL. Tumores malignos de fosas nasales y senos paranasales. In: Suárez Nieto C, editor. Oncología de Cabeza y Cuello, Barcelona: Ars Médica, 2002:75-90.

4. Dulguerov P, Jacobsen M, Allal A, Lehman W, Calcaterra T. Nasal and paranasal sinus carcinoma: are we making progress? A series of 220 patients and systematic review. Cancer 2001;92:3012-3029.

5. Nuñez F, Suárez C, Álvarez I, Losa JL, Barthe P, Fresno MF. Sino-nasal adenocarcinoma: Epidemiological and Clinicopathological study of 34 cases. J Otolaryngol 1993;22:86-90 6. Dulguerov P, Allal AS. Nasal and Paranasal sinus carcinoma: how can we continue to make progress? Current Opin Otolaryngol Head Neck Surg. 2006;14:67-72

7. Konno A, Ishikawa K, Terada N, Numata T, Nagata H, Okamoto Y. Analysis of Long-term Results of Our Combination Therapy for Squamous Cell Cancer of the Maxillary Sinus. Acta Otolaryngol (Stockh) 1998;Suppl 537:57-66

8. Nishino H, Miyata M, Morita M, Ishikawa K, Kanazawa T, Ichimura K. Combined Therapy with Conservative Surgery, Radiotherapy, and Regional Chemoterapy for Maxillary Sinus Carcinoma. Cancer 2000;89:1925-1932.

9. Nibu K, Sugasawa M, Asai M, et al. Results of Multimodality Therapy for Squamous Cell Carcinoma of Maxillary Sinus. Cancer 2002;94:1476-1482.

10. Mannetje A, Kogevinas M, Luce D, et al. Sinonasal cancer, occupation, and tobacco smoking in European women and men. Am J Ind Med. 1999;36:101-107.

11. Götte K and Hörmann K. Sinonasal malignancy: what's new? Review. ORL J Otorhinolaryngol Relat Spec. 2004;66:85-97. 
12. Luce D, Leclerc A, Begin D, et al. Sinonasal cancer and occupational exposures: a pooled analysis of 12 case-control studies. Cancer Causes Control. 2002;13:147-57. 13. Zheng W, McLaughlin JK, Chow WH, Chien HT, Blot WJ. Risk factors for cancers of the nasal cavity and paranasal sinuses among white men in the United States. Am J Epidemiol. 1993;138:965-972.

14. Holt GR. Sinonasal neoplasm and inhaled air toxics. Otolaryngol Head Neck Surg 1994; $11: 12-14$

15. Bandoh N, Hayashi T, Takahara M, et al. VEGF and bFGF expression and microvessel density of maxillary sinus squamous cell carcinoma in relation to p53 status, spontaneous apoptosis and prognosis. Cancer Lett. 2004;208:215-225.

16. Bandoh N, Hayashi T, Kishibe K, et al. Prognostic value of p53 mutations, bax, and spontaneous apoptosis in maxillary sinus squamous cell carcinoma. Cancer. 2002;94:19681980.

17. Holmila R, Cyr D, Luce D, et al. COX-2 and p53 in human sinonasal cancer: COX-2 expression is associated with adenocarcinoma histology and wood-dust exposure. Int $\mathrm{J}$ Cancer. 2008;122:2154-2159.

18. Bornholdt J, Hansen J, Steiniche T, et al. K-ras mutations in sinonasal cancers in relation to wood dust exposure. BMC Cancer. 2008;8:53.

19. Götte K, Riedel F, Schäfer C, Coy J, Hörmann K. Cylindrical cell carcinomas of the paranasal sinuses do not show p53 alterations but loss of heterozygosity at $3 p$ and $17 p$. Int $J$ Cancer. 2000;85:740-742.

20. Gil Z, Orr-Urtreger A, Voskoboinik N, et al. Cytogenetic analysis of sinonasal carcinomas. Otolaryngol Head Neck Surg. 2006;134:654-660.

21. Jin $\mathrm{Y}$, Mertens F, Arheden K, et al. Karyotypic features of malignant tumors of the nasal cavity and paranasal sinuses. Int J Cancer. 1995;60:637-641.

22. Sobin LH, Wittekind C. UICC TNM classification of malignant tumors. 6th ed. New York: Wiley-Liss, 2002. 
23. Guervós MA, Marcos CA, Hermsen M, Nuño AS, Suárez C, Llorente JL. Deletions of N33, STK11 and TP53 are involved in the development of lymph node metastasis in larynx and pharynx carcinomas. Cell Oncol. 2007;29:327-334.

24. Van den ljssel P, Tijssen M, Chin SF, et al. Human and mouse oligonucleotide-based array CGH. Nucleic Acids Res. 2005;33:e192

25. Califano J, van der Riet P, Westra W, et al. Genetic progression model for head and neck cancer: implications for field cancerization. Cancer Res. 1996;56:2488-2492.

26. Hermsen M, Alonso Guervós M, Meijer G, et al. Chromosomal changes in relation to clinical outcome in larynx and pharynx squamous cell carcinoma. Cell Oncol. 2005;27:191198.

27. Snijders AM, Schmidt BL, Fridlyand J, et al. Rare amplicons implicate frequent deregulation of cell fate specification pathways in oral squamous cell carcinoma. Oncogene. $2005 ; 24: 4232-4242$.

28. Järvinen AK, Autio R, Kilpinen S, et al. High-resolution copy number and gene expression microarray analyses of head and neck squamous cell carcinoma cell lines of tongue and larynx. Genes Chromosomes Cancer. 2008;47:500-509.

29. Batsakis JG, Suárez P. Schneiderian papillomas and carcinomas: a review. Adv Anat Pathol. 2001;8:53-64.

30. Caruana SM, Zwiebel N, Cocker R, McCormick SA, Eberle RC, Lazarus P. p53 alteration and human papilloma virus infection in paranasal sinus cancer. Cancer. 1997;79:1320-1328. 31. Califano J, Koch W, Sidransky D, Westra WH. Inverted sinonasal papilloma : a molecular genetic appraisal of its putative status as a Precursor to squamous cell carcinoma. Am $\mathrm{J}$ Pathol. 2000;156:333-337.

32. Singh B, Wreesmann VB, Pfister D, et al. Chromosomal aberrations in patients with head and neck squamous cell carcinoma do not vary based on severity of tobacco/alcohol exposure. BMC Genet. 2002;3:22.

33. Bentires-Alj M, Neel BG. Protein-tyrosine phosphatase 1B is required for HER2/Neuinduced breast cancer. Cancer Res. 2007;67:2420-2424. 
34. Weiss MM, Kuipers EJ, Postma C, et al. Genomic profiling of gastric cancer predicts lymph node status and survival. Oncogene. 2003;22:1872-1879.

35. Akao J, Matsuyama H, Yamamoto Y, Sasaki K, Naito K. Chromosome 20q13.2 gain may predict intravesical recurrence after nephroureterectomy in upper urinary tract urothelial tumors. Clin Cancer Res. 2006;12:7004-7008.

36. Carvalho B, Postma C, Mongera S, et al. Multiple putative oncogenes at the chromosome 20q amplicon contribute to colorectal adenoma to carcinoma progression. Gut. 2009;58:79-89.

37. Astsaturov I, Cohen RB, Harari PM. EGFR-targeting monoclonal antibodies in head and neck cancer. Curr Cancer Drug Targets. 2006 Dec;6(8):691-710. 


\section{FIGURE LEGENDS.}

Figure 1. Example of a MLPA result of case number $27(A)$ and a normal reference DNA (B). Three high peaks in the left panel (see arrows) reflect amplifications of the oncogenes ERBB2, EMS1 and CCND1, respectively.

Figure 2. Example of the maCGH result of case number $26(\mathrm{~A})$. All datapoints are expressed as log2-ratios, ordered continuously from left to right as chromosomes 1 up to chromosomes $X$ and $Y$ (here numbered as 23 and 24). This tumor shows alterations affecting almost all chromosomes. Chromosomes 3 and 19 show high level amplifications (see arrows) and are presented in more detail in panels B and C.

Figure 3. Overview of all DNA copy number gains and losses of 58 different genes as detected by MLPA, expressed in percentages.

Figure 4. Overview of all copy number changes as detected by maCGH. To the right of the pictogram of each chromosome, a scale is placed expressing the number of mega base pairs (Mpb) counting from pter to qter. Copy number losses are presented as bars left to the Mbpscale and copy number gains to the right. 
Table 1. Clinical features of 27 SNSCC patients.

\begin{tabular}{|c|c|c|c|c|c|c|c|c|c|c|c|c|c|}
\hline \multicolumn{3}{|c|}{ Patient } & \multicolumn{5}{|c|}{ Tumor } & \multicolumn{6}{|c|}{ Follow up } \\
\hline $\mathrm{Nr}$ & sex & age & TNM & loc & diff & orbit & IC & RT & rec & met & OS & DFS & status \\
\hline 1 & $M$ & 63 & T4bNOMO & $\max$ & well & 0 & no & yes & no & no & 5 & 5 & A \\
\hline $2^{*}$ & $\mathrm{~F}$ & 50 & T3N0M0 & $\max$ & poor & no & no & no & yes & yes & 77 & 46 & $\mathrm{DOC}$ \\
\hline 3 & $\mathrm{~F}$ & 63 & T4aN0MO & $\max$ & well & 0 & no & yes & yes & yes & 211 & 3 & A \\
\hline 4 & $\mathrm{~F}$ & 69 & T3NOMO & $\max$ & $\mathrm{mod}$ & no & no & yes & no & no & 168 & 60 & $\mathrm{DOC}$ \\
\hline $5^{*}$ & $\mathrm{~F}$ & 72 & T4bNOMO & $\max$ & poor & $\mathrm{PO}$ & yes & no & no & no & 1 & 1 & $\mathrm{DD}$ \\
\hline 6 & $\mathrm{~F}$ & 59 & T3N0M0 & $\max$ & $\mathrm{mod}$ & $\mathrm{PO}$ & no & yes & yes & no & 20 & 6 & $\mathrm{DD}$ \\
\hline 7 & $M$ & 78 & T4aN0M0 & $\max$ & poor & no & no & no & no & no & 7 & 7 & $\mathrm{DD}$ \\
\hline 8 & $M$ & 69 & T4aNOMO & $\max$ & well & no & no & yes & yes & no & 11 & 10 & $\mathrm{DD}$ \\
\hline $9^{*}$ & $\mathrm{~F}$ & 73 & T4aNOMO & $\max$ & poor & 0 & no & no & no & no & 2 & 2 & A \\
\hline 10 & $M$ & 57 & T4aN0M0 & $\max$ & mod & no & no & yes & yes & yes & 27 & 7 & $\mathrm{DD}$ \\
\hline $11^{*}$ & $M$ & 66 & T4aNOMO & $\max$ & $\mathrm{mod}$ & no & no & yes & no & no & 12 & 12 & A \\
\hline 12 & $\mathrm{~F}$ & 74 & T3N0M0 & $\max$ & well & 0 & no & yes & yes & yes & 61 & 9 & $\mathrm{DD}$ \\
\hline $13^{*}$ & $M$ & 49 & T2N0M0 & etm & poor & no & no & yes & yes & no & 13 & 8 & $\mathrm{DD}$ \\
\hline 14 & $\mathrm{~F}$ & 75 & T2NOMO & etm & poor & no & no & yes & no & no & 93 & 93 & A \\
\hline 15 & $M$ & 69 & T4aN0MO & $\max$ & well & no & no & yes & no & no & 90 & 1 & A \\
\hline 16 & $F$ & 53 & T4bNOMO & $\max$ & well & no & no & yes & yes & no & 11 & 8 & $\mathrm{DD}$ \\
\hline 17 & $M$ & 65 & T4aN0M0 & $\max$ & well & $\mathrm{PO}$ & no & yes & yes & no & 55 & 28 & $\mathrm{DD}$ \\
\hline 18 & $M$ & 47 & T4bN0M0 & $\max$ & poor & $\mathrm{PO}$ & no & yes & yes & no & 11 & 11 & $\mathrm{DD}$ \\
\hline 19 & $M$ & 74 & T4aNOMO & $\max$ & well & PO & no & no & yes & no & 4 & 2 & $\mathrm{DD}$ \\
\hline $20^{*}$ & $M$ & 65 & T3N0M0 & $\max$ & poor & no & no & yes & no & no & 82 & 82 & A \\
\hline 21 & $\mathrm{~F}$ & 54 & T4aNOMO & $\max$ & $\mathrm{mod}$ & no & no & yes & yes & no & 18 & 9 & DD \\
\hline $22^{*}$ & $M$ & 64 & T3N0M0 & $\max$ & poor & no & no & no & yes & no & 33 & 12 & $\mathrm{DD}$ \\
\hline $23^{*}$ & $M$ & 68 & T2NOMO & etm & poor & no & no & yes & yes & no & 40 & 32 & A \\
\hline $24^{*}$ & $M$ & 66 & T3N1M0 & etm & well & no & no & yes & yes & no & 16 & 1 & $\mathrm{DD}$ \\
\hline $25^{*}$ & $\mathrm{M}$ & 66 & T3N1M0 & $\max$ & well & no & no & yes & yes & no & 8 & 2 & $\mathrm{DD}$ \\
\hline $26^{*}$ & $M$ & 85 & T4aN1M0 & $\max$ & poor & 0 & no & no & no & no & 1 & 1 & $\mathrm{DOC}$ \\
\hline 27 & $\mathrm{M}$ & 71 & T4aN1M0 & $\max$ & $\mathrm{mod}$ & no & no & no & yes & yes & 6 & 4 & $\mathrm{DD}$ \\
\hline
\end{tabular}

Legend. sex; F: female, M: male; age: in years; TNM: tumor stage according to the IUCC; loc: localization; max: maxillary sinus; etm: ethmoid sinus; diff: grade of differentiation; mod: moderate; orbit: invasion into orbit; O: orbit; PO: periorbit; IC: intracranial invasion; RT: radiotherapy; rec: local recurrence; met: distant metastasis; OS: overall survival (months); DFS: disease-free survival (months); A: alive; DD: died of disease; DOC: died of other causes. The 11 cases analyzed with microarray $\mathrm{CGH}$ are marked with an asterisk. 
Table 2a. High level amplifications as detected by maCGH.

\begin{tabular}{|c|c|c|c|}
\hline Chromosomal band & Mbp & Candidate genes & cases \\
\hline $1 p 12$ & $118.1-120.0$ & NOTCH2 & 22 \\
\hline $3 q 26.1-3 q 28$ & $165.8-189.4$ & PIK3CA & 26 \\
\hline $5 p 15.3$ & $0.65-0.97$ & $?$ & 25 \\
\hline $7 p 12$ & $54.4-55.1$ & ERBB1 (EGFR) & $23,24,26$ \\
\hline $8 q 24.3$ & $145.7-145.9$ & RECQL4, PTP4A3 & 25 \\
\hline $11 p 13$ & $34.1-36.2$ & CD44 & 9,24 \\
\hline $11 p 12$ & $40.0-44.2$ & $?$ & 26 \\
\hline $11 q 13.2-11 q 14.1$ & $68.5-82.2$ & CCND1, EMS1 & 13 \\
\hline $11 q 21-11 q 22.1$ & $95.9-102.9$ & BIRC2, YAP1 & 5 \\
\hline $12 q 13.1$ & $44.3-46.7$ & $?$ & 22 \\
\hline $17 q 21$ & $33.8-35.2$ & ERBB2 (Her2/neu) & 22 \\
\hline $19 p 13.2$ & $12.1-14.7$ & JUNB & 22 \\
\hline $19 q 13.2$ & $45.0-45.6$ & AKT2, MAP3K10 & 26 \\
\hline $19 q 13.3$ & $49.6-50.8$ & $?$ & 22 \\
\hline
\end{tabular}

Legend. The amplifications are localized in the chromosomal bands given in the first column, but can be expressed more precisely in mega base pairs (Mbp; positioning of oligos according to NCBI Build 36.1, March 2006). Possible oncogenes present within these amplicons are suggested, but does not seek to be complete. 
Table 2b. High level amplifications as detected by MLPA.

\begin{tabular}{|c|c|c|}
\hline Chromosomal band & Gene & cases \\
\hline $1 \mathrm{p} 21$ & LMNA & 1 \\
\hline $3 \mathrm{q} 26$ & PIK3CA & 6 \\
\hline $7 \mathrm{q} 21$ & ABCB1 & 27 \\
\hline $8 \mathrm{q} 24$ & CMYC & 4 \\
\hline $8 \mathrm{q} 24$ & RECQL4, PTP4A3 & 25 \\
\hline $11 \mathrm{q} 13$ & EMS1,SSND1 & $3,4,13,27$ \\
\hline $17 \mathrm{q} 21$ & ERBB2 & 22,27 \\
\hline $19 \mathrm{p} 13$ & CDKN2D, STK11 & 1 \\
\hline
\end{tabular}


Table 3. Correlation of gene copy number gains with tumor dissemination.

\begin{tabular}{|l|c|c|c|}
\hline & \multicolumn{2}{|c|}{ lymph node or distant metastasis } & \\
\hline & No $(\mathrm{n}=19)$ & Yes $(\mathrm{n}=8)$ & Fisher Exact \\
\hline gain of PTPN1 & 1 & 5 & $\mathrm{p}=0.004$ \\
\hline gain of BCL2L1 & 5 & 5 & $\mathrm{p}=0.091$ \\
\hline gain of CCND1 & 3 & 4 & $\mathrm{p}=0.088$ \\
\hline gain of EMS1 & 5 & 5 & $\mathrm{p}=0.091$ \\
\hline gain of PTP4A3 & 1 & 3 & $\mathrm{p}=0.065$ \\
\hline
\end{tabular}

